\title{
Identification of Safe Zone with Intraoperative Neurophysiological Monitoring during Surgical Removal of Peripheral Nerve Tumor
}

\author{
Dong-hun Kim ${ }^{1}$, Jin-gyu Choi ${ }^{1}$, Byung-chul Son ${ }^{1,2}$ \\ ${ }^{l}$ Department of Neurosurgery, Seoul St. Mary's Hospital, College of Medicine, The Catholic University of Korea, Seoul, \\ ${ }^{2}$ The Catholic Neuroscience Institute, College of Medicine, The Catholic University of Korea, Seoul, Korea
}

\begin{abstract}
During resection of peripheral nerve tumor, it is essential to identify and protect the neighboring neural elements to the tumor itself prior to attempting direct resection. The authors present the techniques of intraoperative electrophysiologic monitoring and nerve stimulation during resection of schwannoma of the peripheral nerve tumor in the upper extremity. After performing a longitudinal epineurotomy in a 'bare area' of the tumor (devoid of fascicular structure), an intraoperative bipolar nerve stimulation was given $(0.5-1 \mathrm{msec}, 1 \mathrm{~mA})$ to identify safe zones to assist to determine functioning and nonfunctioning fascicles, and therefore facilitate resection. With the aid of microscopic dissection and intraoperative nerve stimulation, the schwannoma could be removed by opening the epineurium at a 'bare area' and separating the uninvolved fascicles until the single feeding fascicle was identified. Intraoperative neurophysiology and nerve stimulation is a helpful technique to facilitate complete removal of peripheral nerve tumor unassociated with neurologic deficit.
\end{abstract}

Key Words: Intraoperative neurophysiological monitoring $\cdot$ Electric stimulation $\cdot$ Peripheral nerves $\cdot$ Neurilemmoma

\section{INTRODUCTION}

Surgery is successful in preserving function in $90 \%$ of schwannomas $^{1)}$. An essential goal of the surgery is the preservation of a maximum of the undamaged nerve fascicles, that is, not sacrificing healthy nerves, and it is in this aspect that intraoperative neurophysiological monitoring (INM) studies are of great value, as they provide the surgical team with basic, reliable and real-time information on the functionality of the explored nerve $\mathrm{e}^{6-8)}$. The authors present an intraoperative, microstimulation technique to identify "safe zone" which is devoid of neural fascicular structure during removal of schwannoma involving the peripheral nerve.

\section{CASE REPORT}

\section{History and Examination}

A 29-year-old male patient presented a slowly growing pal-

Corresponding author: Byung-chul Son

Department of Neurosurgery, Seoul St. Mary's Hospital, The Catholic Neuroscience Institute, College of Medicine, The Catholic University of Korea, 222 Banpo-daero, Seocho-gu, Seoul 06591, Korea Tel: +82-2-2258-6122, Fax: +82-2-594-4248

E-mail: sbc@catholic.ac.kr pable mass in his right medial axilla for 3 years of duration with paresthesia in right second finger (Fig. 1A). The size of the mass was about $2 \mathrm{~cm}$, and it was movable, well-marginated with rubbery consistency. A manipulation of the mass typically elicited a severe paresthesia in the distal part of the second finger. No motor weakness or sensory disturbance was noted and there was no other sign of skin pigmentation such as caféau-lait spots.

An ultrasonographic examination showed a heterogeneously hypoechoic mass separated from neighboring nerve and vessels (Fig. 1B). In the magenetic resonance imaging (MRI) study, it showed slightly high signal intensity on T2-weighted images (WI) and a low signal intensity on T1-WI (Fig. 1C). A strong gadolinium enhancement was noted (Fig. 1C). The mass was well localized within neurovascular bundles in the proximal upper arm, just distally to the brachial plexus. Considering the growing nature of the mass with paresthesia and radiological findings, a surgical resection was planned under the provisional diagnosis of the schwannoma involving the high median nerve just distal to the brachial plexus.

\section{Operation under INM}

Under general anesthesia, the patient was place in the supine position with the right arm extended and the head rotated to the opposite side. About 10-cm-sized incision was made over the palpable mass in medial axillary region. After dissect- 


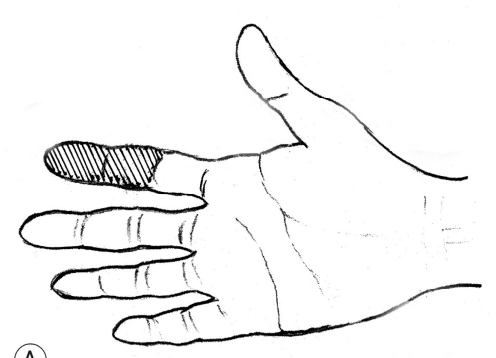

(A)
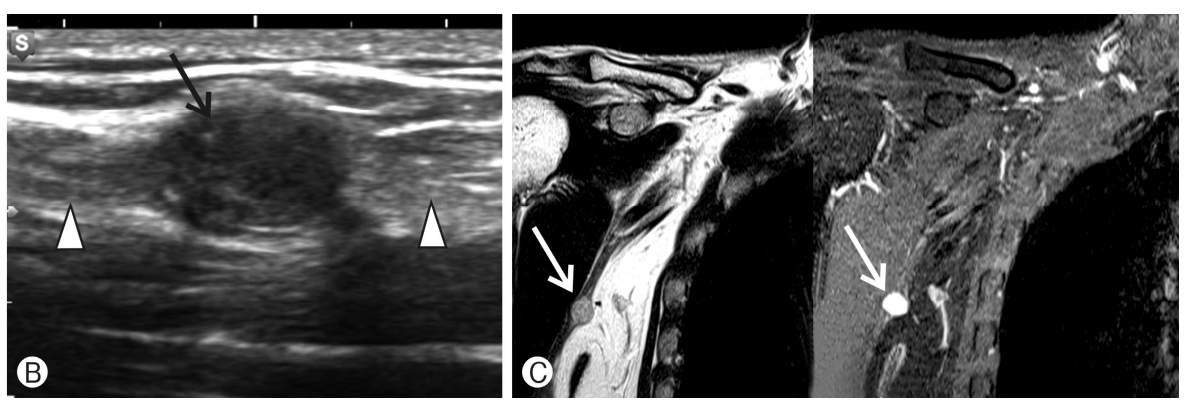

Fig. 1. Distribution of paresthesia and radiologic findings of the patient. (A) The hatched area shows the distribution of typical paresthesia in the distal portion of the second finger. (B) A longitudinal ultrasound scan image shows a round, deeply hypoechoic mass (arrow) with a hypoechoic nerve fascicles (white arrowheads). (C) A T2-weighted coronal magnetic resonance imaging (MRI) shows a slightly hypointense, small round mass (arrow) along the high median nerve (left) and a strong gadolinium enhancement was noted in T1-weighted coronal MRI image.
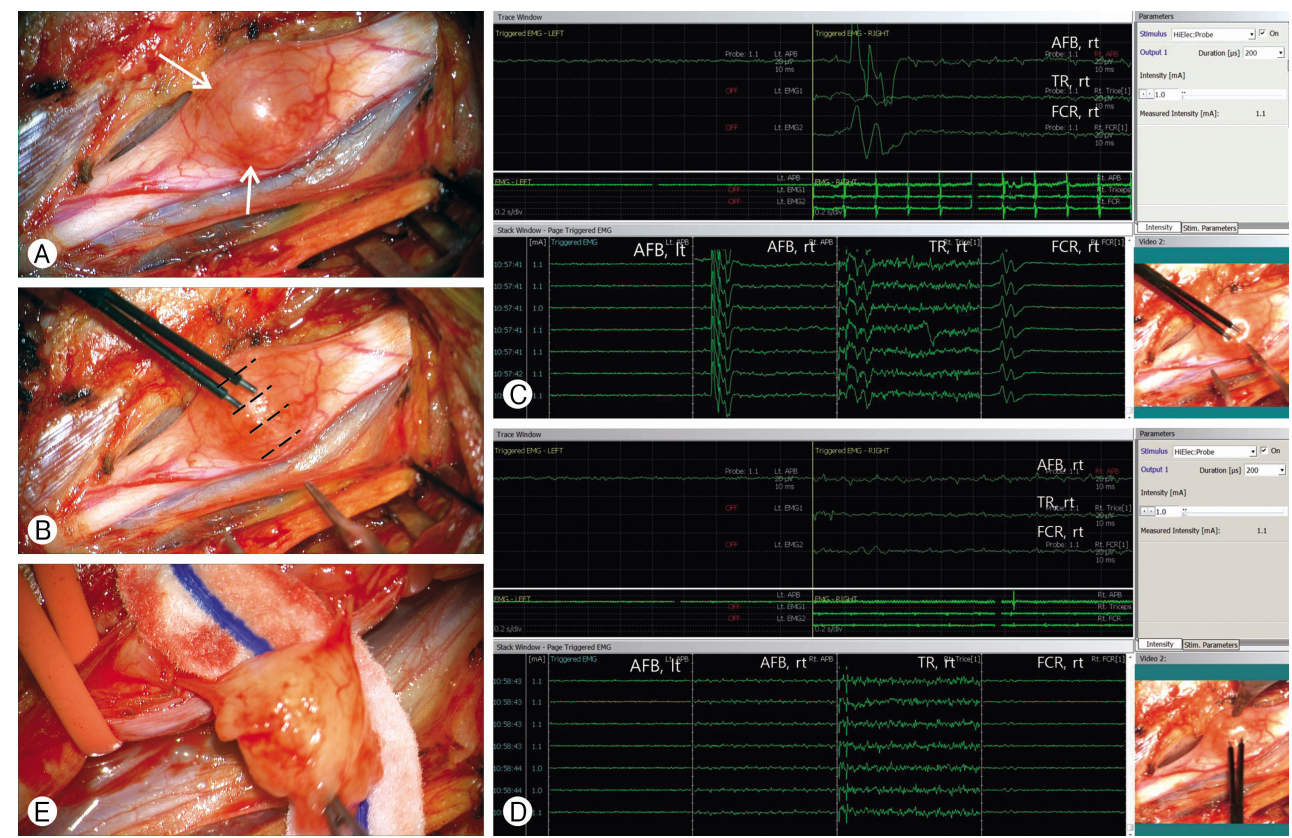

Fig. 2. Intraoperative photographs showing the removal of schwannoma with a guidance of intraoperative bipolar stimulation and evoked electromyography (EMG) responses. (A) An intraoperative photograph showing the exposure of the tumor (arrows) in the high median nerve in the right axillary region. (B) An intraoperative photograph showing the bipolar stimulation of the tumor to find the safe zone for dissection. The stimulation $(0.5-1 \mathrm{msec}, 1 \mathrm{~mA})$ is given several points (white lines) over the tumor surface, along the course of the nerve fascicles. (C) An evoked EMG response from bipolar stimulation of the nerve fascicle-containing area over the tumor surface. Note the prominent EMG activity in the abductor pollicis brevis (APB). FCR: flexor carpi radialis; TR: triceps. (D) An evoked EMG response from bipolar stimulation of the silent area (safe zone) which is devoid of nerve fascicle. Note an electric silence in the right APB (arrow). (E) An intraoperative photograph showing the final stage of dissection of the nerve fascicle directly involved in the tumor. This fascicle involving the tumor was removed en-bloc.

ing the subcutaneous fat tissue and fascia, soft tissue was removed around the bulging mass. The proximal median nerve, the distal part of brachial plexus, was isolated under the microscopic vision (Fig. 2A).
After performing a longitudinal epineurotomy in the median nerve, a bipolar stimulation $(0.5-1 \mathrm{msec}, 1 \mathrm{~mA})$ over the several points of the bulging median nerve was performed to map the "safe zone" of the tumor (Fig. 2B). "Safe zones" 
was confirmed, if contraction of the distal muscle had not been occurred by neurophysiologic stimulation (Fig. 2C, D). Marking the lines along the safe zones, the fine dissection under microscopic vision had been performed until the involved fascicle was exposed. The involved fascicle to the tumor was finally isolated and resected en-bloc (Fig. 2E).

\section{Postoperative Course}

Following en-bloc resection of the mass, he reported a slight hypoesthesia and paresthesia in the distal portion of the right $2^{\text {nd }}$ finger. No motor weakness was noted. The hypesthesia and paresthesia progressively improved over the 2 months and eventually no sensory deficit was observed in 3 months postoperatively. The pathologic examination revealed that the mass was a schwannoma. The tumor was positively stained to S-100, negative for epithelial membrane antigen (EMA), and had a low Ki-67 index (9\%).

\section{DISCUSSION}

Schwannomas can be numerous, but tends to be solitary. Solitary schwannoma that are enucleated very rarely recur, and malignant transformation in a solitary schwannoma has been reported in only 12 cases in the literature ${ }^{1)}$. Nevertheless, treatment is largely based on surgical removal except for asymptomatic schwannoma. Removal of the tumor with minimization of residual neurologic deficit is the goal of surgery ${ }^{2}$. In this respect, INM studies are invaluable, by providing the surgical team with critical information for minimal invasive surgery in order to preserve the function of the involved nerve ${ }^{3)}$.

A retrospective review reported in a representative sample of 76 patients with schwannomas, baseline function was preserved or improved $89 \%$ of the time ${ }^{4)}$. Another study compared the effectiveness of surgical treatment by grading the preoperative and postoperative motor function (0-5). Ten point five percent of patients presented post-operative motor weakness after surgery ${ }^{5}$. However, in a recent report ${ }^{3)}$ in 2014 involving 12 cases of peripheral nerve schwannomas, 9 in the upper limbs and 3 in the lower limbs, the surgery was carried out with no incidents and the tumor was resected from the main trunk of the nerve, except for 1 case where we had to sacrifice an only-sensory branch of the posterior tibial nerve after the neuro-physiological monitoring showed that it had no motor component ${ }^{3)}$.

In our case, the patient had the typical history and examination results consistent to the benign schwannoma. We needed to remove the tumor minimally invasively, and adopted a technique of intra-operative neurophysiologic monitoring for the minimum loss of neurologic deficit. As a result, we could remove the schwannoma without any long-term deficit. However, despite of INM, it is impossible to clarify the composition of the tumor, and the possibility of malignancy. There are also some limitations in order to predict exactly if the patients will have a dysesthesia or pain.

\section{CONCLUSION}

An intraoperative neurophysiology and nerve stimulation is a useful technique to facilitate complete removal of peripheral nerve tumor and to preserve functioning nerve fascicles.

\section{REFERENCES}

1. Barbour JR, Boyd KU. Tumors of the peripheral nervous system. In: Mackinnon SE, Yee A eds. Nerve Surgery. New York: Thieme, 2015, pp530-571

2. Golan JD, Jacques L: Nonneoplastic peripheral nerve tumors. Neurosurg Clin N Am 15:223-230, 2004

3. Herrera-Pérez M, Oller-Boix A, Pérez-Lorensu PJ, de BerguaDomingo J, Gonzalez-Casamayor S, Márquez-Marfil F, et al.: Intraoperative neurophysiological monitoring in peripheral nerve surgery: Technical description and experience in a centre. Rev Esp Cir Ortop Traumatol 59:266-274, 2015

4. Kim DH, Murovic JA, Tiel RL, Moes G, Kline DG: A series of 397 peripheral neural sheath tumors: 30-year experience at Louisiana State University Health Sciences Center. J Neurosurg 102:246-255, 2005

5. Kline DG, Hudson AR, Tiel RL, Guha A. Management of peripheral nerve tumors. In: Winn HR ed. Youmans Neurological Surgery, ed 5th. Philadelphia: Saunders, 2004, pp3941-3957

6. Kwok K, Davis B, Kliot M: Resection of a benign brachial plexus nerve sheath tumor using intraoperative electrophysiological monitoring. Neurosurgery 60:316-321, 2007

7. Mrugala MM, Batchelor TT, Plotkin SR: Peripheral and cranial nerve sheath tumors. Curr Opin Neurol 18:604-610, 2005

8. Pilavaki M, Chourmouzi D, Kiziridou A, Skordalaki A, Zarampoukas T, Drevelengas A: Imaging of peripheral nerve sheath tumors with pathologic correlation: pictorial review. Eur J Radiol 52:229-239, 2004 